\title{
Jahres-Inhalt, Vol. 10, №. 6, 1987
}

\section{Editorial 4}

Pralle, H., Gießen, et al.

Primäre Behandlung der Haarzelleukämie mit niedrigdosiertem humanem rekombinantem Interferon-Alpha2c (Hr-IFn Alpha 2c) im Vergleich zur Therapie nach Splenektomie. - Interferon (IFn-Alpha 2c) bei HCL vor oder nach Splenektomie 5

Erratum 10

Hiddemann, W., Gießen, et al.

Behandlung refraktärer akuter lymphoblastischer Leukämien mit Hoch-Dosis Cytosin Arabinosid und Mitoxantron (HAM) 11 Büchner, Th., Münster, et al.

Alternativen und Weiterentwicklung der Therapie der akuten myeloischen Leukämie (AML) des Erwachsenen. -Update der bundesdeutschen multizentrischen Studien . . 14

Maschmeyer,G.; Kreutzmann,H. und Wendt,F., Essen; Hiddemann, W., Münster

Chemotherapie der akuten nicht-lymphatischen Leuk

ämie bei Hochrisikopatienten unter Verwendung von

m-Amsacrine 18

Leserzuschrift 22

Buchbesprechungen 22

Ritter, J., Münster, et al.

Hochdosiertes ARA-C in Kombination mit Mitoxantron bei der Therapie der AML im Kindesalter. - Erste Ergebnisse der AML-Rezidivstudie BFM-85 24

Hehlmann, R., München, et al.

Prospektive kontrollierte Studie zur Therapie der chronisch myeloischen Leukämie (CML) 28

Köppler, H., Marburg, et al.

Multizentrische Studie zur Behandlung hochmaligner Non-Hodgkin-Lymphome mit Polychemotherapie (CHOPV) und Bestrahlung 34 Informationen für die Klinik 42 Messerer, D.; Hasford, J. und Neiß, A., München; Porzsolt, F, Ulm Vorteile und Probleme multizentrischer Therapiestudien am Beispiel einer Studie zur Behandlung des metastasierenden Nierenzellkarzinoms mit rekombinantem Inter feron-Alpha-2c 43 Kern, P., Hamburg, et al. 
Interferon Alpha in der Behandlung des AIDS-assoziier-

ten Kaposi-Sarkoms 50

Blöchl-Daum, B., Wien/Österreich, et al.

Phase-I-Studie: Intraperitoneales Mitoxantron - Klini-

sche und pharmakokinetische Auswertung 54

Dennig, D.; Fülle, H.H. und Hellriegel, K.-P., Berlin

Chemoprophylaxe bakterieller Infektionen mit Cipro-

floxacin bei granulozytopenischen Patienten 57

Diehl, V.'; Pfreundschuh, M.; Löfflet; M. und Smith, K., Köln; Rühl, U., Berlin; Georgii, A., Hannover; Hiller, E. und Gerhartz, H., München

Therapiestudien der deutschen Hodgkin-Studiengruppe. -

Zwischenergebnisse der StudienprotokoUe HD1, HD2

undHD3 62

Flechtner, H.; Queißer, W. undHeím, M. E., Mannheim; Henß, H. und Arnold, H., Freiburg; Fritz,e, D., Darmstadt; Herrmann, R. und Endler, L., Heidelberg; Fritsch, H., Weinheim; Fritz, M., Karlsruhe; Trux, F.A., Schweinfurt; Kabeliiz, K., Homburg 5-Fluorouracil , 4Epidoxorubicin und Mitomycin C (FEM) Kombinationschemotherapie beim fortgeschritte-nen Magenkarzinom. - Eine Phase-II-Studie der «Chemo-therapiegruppe Gastrointestinaler Tumoren (CGT)» ... 67

380

Jahres-Inhalt 1987

Reiner, G.; Reiner, A.; Jakesz, R- und Kolb, R.,

Wien/Österreich

Wertigkeit der Schnellschnittuntersuchung zur intra-

operativen Beurteilung des axillären Lymphknotenbefalls

beim Mammakarzinom 72

Geyer, H. und Braun, A., Freiburg

Kernrezeptoren in Östrogenrezeptor-negativen Tumoren

mit Progesteronrezeptoren 79

Brambs, H.-J.; Freund, U.; Bruggmoser, G. und Wannenmacher, M., Freiburg

Kombinierte intraduktale und perkutane Radiotherapie

bei maligner Gallenwegsobstruktion mit anschließender

prothetischer Versorgung 84

Koschel, G.; Kaukel, E. und Hain, E., Hamburg

Chemotherapie des nicht-kleinzelligen Bronchialkarzi-

noms mit Mitomycin C, Vindesin und Cisplatin. - Ergeb-

nisse einer Phase-II-Studie 90

Ammon, A. und Graewe, T., Göttingen

Kasuistischer Beitrag zur immunzytochemischen Diffe-

rentialdiagnostik von serösen Ergüssen und Zysten-

flüssigkeit $\quad 97$

Baumgartner, G.; Horaczek, A.; Grunert, P.; Kitz,

K. und Wunsch, M., Wien/Österreich

Hyaluronidase als Zusatz zur zytostatischen Chemothera

pie bei Glioblastomen 100 
Wander, H.-E.; Nagel, G.A. und Bönisch, E., Göttingen; Kleeberg, U.R., Hamburg; Gartner, E., Hildesheím; Hartlapp, J., Bonn; Scherpe, A., Stade Megestrolazet versus

Medroxyprogesteronazetat in der Behandlung metastasierender Mammakarzinome. - Zwischenbericht einer multizentrischen Phase-III-Studie . . . 104

Editorial 112

Diehl, V. und Pfreundschuh, M., Köln

Klinische Relevanz der Immuntypisierung onkologischer

und hämatologischer Erkrankungen 114

Emmrich, F., Erlangen

Perspektiven der Anwendung monoklonaler Antikörper

in der Therapie neoplastischer Erkrankungen 121

Bartram, C.R., Ulm

Molekulargenetische Untersuchungen zur Pathogenese

und Klassifizierung der chronísch myeloischen Leukämie . 127

Pandolfi, F.; Cafaro, A. und Scarselli, E., Romlltalien

Relevanz der Phänotypisierung von monoklonalen Anti-

körpern und gentechnischer Studien bei der Klassifizie

rung von T-Zell-Leukämie/Lymphomen 134

Kleihauer, E., Ulm

Diagnostik und Therapieansätze mit gentechnologischen

Methoden bei Thalassämien 138

Fauser, A. A. und How son-Jan, K., Montreal/Kanada;

Kanz, B. und Löhr, G. W., Freiburg

Immunregulation der Hämatopoese 144

Nissen, C, Basel/Schweiz

153

Serumfaktoren und deren Einfluß auf die Hämatopoese

Müller, C.A.; Busch, F. W. und Waller, H.D., Tubingen

Bedeutung des HLA-Systems in der Regulation der

Hämatopoese 157

Storb, R. und Witherspoon, R., Seattle, WA./USA

Welche Faktoren entscheiden über Erfolg oder Mißerfolg

der Knochenmarktransplantation? 166

108

Informationen für die Klinik 107

Buchbesprechungen

Bacigalupo, A.; Piaggio, G.; van Lint, M. T.; Figari, O.;

Marmont, A. und Congiu, M., Genua/Italien

Aplastische Anämie: Pathogenese und Behandlung. . . . 172

Jahres-Inhalt 1987

381

Niethammer, D., Tubingen

Störungen der Hämatopoese bei Immundefekten

Informationen für die Klinik 180 
Editorial 184

Kurth, R.; Lower, R. und Lower, /., Frankfurt;

Gelderblom, H., Berlin

Retroviren und Lymphomentstehung 186

Bornkamm, G. W.; Polack, A.; Eick, D.

und Berger, R., Freiburg; Lenoir, G., LyonlFrankreich

Chromosomentranslokationen und Epstein-Barr-Virus

beim Burkitt-Lymphom 196

Fonatsch, Ch. und Gradl, G., Lílibeck Chromosomenaberrationen und maligne Lymphome . . .

206

Ehninger, G.; Schuler, U. und Waller, H.D., Tubingen

Pharmakokinetische Aspekte beim Einsatz neuer Zyto-

statika 218

Gallmeier, W.M. und Bruntsch, U., Nürnberg

Neue Zytostatika - Stand: $1986 \quad 226$

Schwendener, R.A.; Supersaxo, A.; Rubas, W.

und Hengartner, H., Zurich!Schweiz;

Schott, H., Tubingen; Hartmann, H.R., Basel/Schweiz

Liposomen als Träger von lipophilen Cytosinarabinosid-

und Fluorodeoxyuridin-Derivaten - Ihre zytostatische

Wirkung und Möglichkeiten zur tumorzellspezifischen

Therapie 232

Kalden, J.R., Erlangen

Zytokine in der Tumortherapie $\quad 240$

Gall, F. P., Erlangen

Die Resektion von Lebermetastasen 247

Franke, R.P.; Höpken, S.; Schnittler, H.;

Dauer, U.; Zangs, R. und Mittermayer, Ch., Aachen;

Kiesewetter, H., Homburg

Endothelfunktion und Arteriosklerose $\quad 250$

Nawroth, P.P. und Stern, D.M., Oklohama City/USA

Veränderung der Gerinnungseigenschaften des Endo-

thels durch Tumor Nekrosis-Faktor 254

Sonderbände 258

Risau, W., Tubingen

Endothel, Angiogenese und Metastasierung 260

Informationen für die Klinik 263

Zacharski, L.R., Vermont/USA

Kleinzelliges Bronchialkarzinom: Interaktionen mit dem

Gerinnungssystem und Behandlung mit Antikoagulan-

zien 264

Abstrakts zur Gemeinsamen Frühjahrstagung der Osterreichischen Gesellschaft für Hämatologie und Onkologie und der Osterreichischen Gesellschaft für Allergologie undlmmunologie, 27.

März 1987, Wien/Österreich . . . . 274

Sonderbände 283

Herbolsheimer, M.; Richter, E. und Schmitt, R., Würz- 
burg; Romen, W., Bad Mergentheim

Bericht über ein Fünffachmalignom 284

Jäger, W. und Sauerbrei, W., Erlangen; Diedrich, M.; Krempel, B. und Wildt, L., Bonn

CA-15/3-Serumkonzentrationen während 24 Stunden bei

Patientinnen mit primären und metastasiertem Mamma-

karzinom 290

Sauer, H.; Kremer, G. und Wilmanns, W., München

Zur Toxizität einer CYVADIC-Modifikation bei Patien-

ten mit Weichteilsarkomen oder malignen Mesothelio-

men 294

Stuller, I.; Zielinski, C. C; Aiginger, P.; Rausch, P.;

Pötzi, P.; Kubista, E.; Salzer, H.; Sevelda, P.;

Longer, M.; Staff en, A. und Spona, ]., Wien/Österreich

Der Einfluß von Hormonrezeptoren auf die Metastasen-

lokalisation beim Mammakarzinom 302

382

Jahres-Inhalt 1987

Informationen für die Klinik 304

Fricke, J.; Krasemann, E.O. und Fehr, R., Wedel Regionale Verteilung der Sterbefälle von

Krebskranken im Hamburger Stadtgebiet 1970-1972 und deren Vergleich mit der Verteilung in den Jahren 1960-1962 … 305

Battegay, M.; Obrist, R. und Obrecht, J.P., Basel/

Schweiz

10 Jahre Therapie des M. Hodgkin an der Onkologischen

Abteilung des Kantonsspitals Basel: Eine retrospektive

Analyse $\quad 308$

Jehn, U.; Vehling-Kaiser, U.; Jaspers, L. und

Göldel, N., München

Gleichzeitiges Auftreten einer akuten myeloischen Leuk-

ämie (AML) bei zwei Chemiearbeitern desselben Werkes 312

Buchbesprechungen 316

Wander, H.-E.; Blossey, H. C. und Nagel, G.A.,

Göttingen; Kleeberg, U.R., Hamburg;

Essers, U., Aachen

Aminoglutethimid plus hochdosiertes Medroxyproge-

steronazetat versus Aminoglutethimid plus Kortison in

der Therapie des metastasierenden Mammakarzinoms.

Eine AIO-Phase-II-Studie 321

Renner, D.; Queißer, U.; Martinez, C. und

Queißer, W., Mannheim

Behandlung der exzessíven Thrombozythämie bei chroni-

scher myeloischer Leukämie mit Thrombozytopherese

und intravenöser Chemotherapie mit Thio-TEPA 324

Edler, L. und Flechtner, H., Heidelberg

Remission in Phase-II- und Phase-III-Studien: Kriterien 
und Voraussetzungen 330

Doberauer, C; Niederle, N.; Kloke, O.; Kurschel, E. und Schmidt, C.G., Essen

Zur Behandlung des metastasierten Karzinoids von Ileum und Caecum mit rekombinantem

Interferon alpha-2b. . . 340

Heím, M. E., Mannheim; Fritze, D., Darmstadt; Ho, A. D., Heidelberg; Mebes, W., Mannheim; Abel, U., Heidelberg Phase-III-Studie zur Chemotherapie niedrig-maligner Non-Hodgkin-Lymphome: Vergleich einer Vincristin- mit einer Vindestin-Kombinations-Chemotherapie $\quad 345$ Hermann, M.; Kober, F. und Keminger, K., Wien Die Fernmetastasierung der Struma maligna. Eine retro spektive Studie über 892 Fälle $\quad 350$ Informationen für die Klinik 356 Wolf, M. und Havemann, K., Marburg; Holle, R. und Drings, P., Heidelberg; Hans, K., Oberhausen;

Schroeder, M., Duisburg

Rezidivhäufigkeit und Langzeit-Überleben beim kleinzelligen Bronchialkarzinom 357

Sonderbände 366

Porzsolt, F. und Schreml, W., Ulm; Buchelt, E., Göppingen; Meuret, G. und Mende, S., Ravensburg;

Strigl, P., Schwäbisch Hall; Redenbacher, M., Schwäbisch Gmünd; Klumpp, D., Heidenheim; Schmelz, M., Niirtingen; Knöchelmann, R., Filderstadt; Hiemeyer, V., Kempten; Fleischer, K., Geislingen; Kreuser, E.-D.; Eeichtle, R.; Popp, C. und Kloiber, R., Ulm Konzept zur Behandlung metastasierender Mammakarzinome außerhalb von Universitätskliniken: Beschreibung der Methode und Prüfung der Effizienz 367

Buchbesprechungen 374

Drings, P., Heidelberg; Calcanis, A., Grenz,ach

Furtulon (Doxifluridin) in der Behandlung des nichtkleinzelligen Bronchialkarzinoms 376

Jahres-Inhalt $1987 \quad 379$

Autorenverzeichnis $1987 \quad 383$

Supplement 1/Februar 1987

Mammakarzinome - Empfehlungen zur Diagnostik, The rapie und Nachsorge (Projektgruppe «Mammakarzinom» im Tumorzentrum München) 1-44 